\title{
Structure of High Transparent Polypropylene Film
}

\author{
Mitsuhiro Shibayama, Kuniyashu Katoh, ${ }^{* 1}$ Takehiro Iwamoto, ${ }^{* 2}$ \\ Daisuke TaKaHAShI, ${ }^{* 3}$ and Shunji Nomura \\ Department of Polymer Science and Engineering, Kyoto Institute of Technology, \\ Matsugasaki, Sakyo-ku, Kyoto 606, Japan
}

(Received October 5, 1990)

\begin{abstract}
We investigated the structure of highly transparent polypropylene (PP) films by ultraviolet-visible (UV-VS) light spectroscopy, light scattering, and polarized optical microscopy. Two kinds of films, PRSQ (sampled after quenching with flowing water) and PRSA (sampled after roll annealing process), were prepared by Idemitsu Petrochemical Co., Ltd., Japan, with a technique which included a rapid quenching with flowing cold water followed by a roll annealing process. A reference film, PPR (a PP film prepared without water cooling process but cooled with a chill-roll kept at $60-80^{\circ} \mathrm{C}$ ) was also prepared by the company. The turbidity, $\tau=\log \left(I_{0} / I\right) / t$, was measured for PRSQ, PRSA, and PPR, where $I_{0}, I$, and $t$ are the intensities of the incident light and the transmitted light, and the sample thickness, respectively. The values of turbidity at the wavelength of $632.8 \mathrm{~nm}$, which is far from the characteristic absorption bands of polypropylene, were 0.35 , 0.14 , and $5.00 \mathrm{~cm}^{-1}$, respectively for PRSQ, PRSA, and PPR. The lowering of the turbidity, in another word, the increase in transparency, of PP films by annealing is explained with lowering of optical anisotropy in a spherulite by formation of crosshatched crystals and lowering of density difference between spherulites and matrix.
\end{abstract}

KEY WORDS Polypropylene / Transparency / Turbidity / Spherulite / Sheaf-Like Structure / Depth Profiling /

A large amount of plastic films is used for wrapping materials which require the following properties, e.g., high gas barrier, heat resistance, appropriate thermal and mechanical strength, and transparency. Among them, transparency depends on the surface and the internal structure of the material which is formed during its solidification process. Particularly, in the case of crystalline polymer films, an improvement of transparency has been rather difficult due to crystallization although those films have superior mechanical properties compared to amorphous polymer films.

In general, transparency of crystalline poly- mers is reduced by presence of spherulites. Transparency is usually raised by (1) quenching, (2) adding a nucleating reagent, or (3) destroying the spherulites by either uniaxial or biaxial stretching. However, the second and third methods are not appropriate for food and drug wrapping since contamination of the nucleating reagent and shrinking of the film during the processing, respectively, are highly expected. It has been believed that quenching is not effective enough to raise the transparency of films for commercial use.

Among a large number of crystalline polymers, polypropylene films have several advantages; relatively high melting point, weather

Present address:

*1 Central Research Laboratory, Nippon Gosei Co., Ltd., Ibaraki, Osaka 567, Japan.

*2 Packaging Products $R \&$ D Center, Plastics Products Department, Idemitsu Petrochemical Co., Ltd., Himeji 672, Japan.

*3 Rakuyo Kogyo High School, Minami-ku, Kyoto 601, Japan. 
and mechanical durability, production cost, and processability. Particularly the high heat resistance has extended the market of food wrapping materials since it enabled one to sterilize them at a high temperature.

Five types of crystalline structures for polypropylene, ${ }^{1-3} \alpha,{ }^{4} \beta,{ }^{5-7} \gamma, \delta$, and smectic structures, were reported in the literature. Among them, monoclinic and smectic structures are often observed in film processing. Keith et al. ${ }^{8}$ classified spherulite structures of PP into five categories according to their crystal structures and birefringence. Crystalline structures in a spherulite were discussed by Bassette et al. ${ }^{9}$ and by Norton. ${ }^{10}$

Idemitsu Petrochemical Co., Ltd., however, recently developed a method to obtain a highly transparent polypropylene (PP) films by quenching molten PP with flowing water followed by roll annealing. ${ }^{11}$ By using this technique, transparency of PP films was highly improved compared with conventional methods.

In the previous paper, ${ }^{12}$ we reported the turbidity change of PP films by recycling of the film preparation. Turbidity increased with the number of recycles, which resulted from formation of spherulites near the film surface. This phenomenon was explained as follows: Once spherulites were formed, they became seeds for crystallization in the next cycle of the preparation, thus the number of the spherulites increased by recycling processes.

We discuss here the structure of high transparent PP films by a turbidity measurement with ultraviolet and visible light spectroscopy, light scattering, densitometry, and polarized optical microscopy. The thermal properties, including thermal history dependence of transparency, will be reported in the forthcoming paper. ${ }^{13}$

\section{EXPERIMENTAL}

\section{Samples}

High transparent polypropylene (PP) films

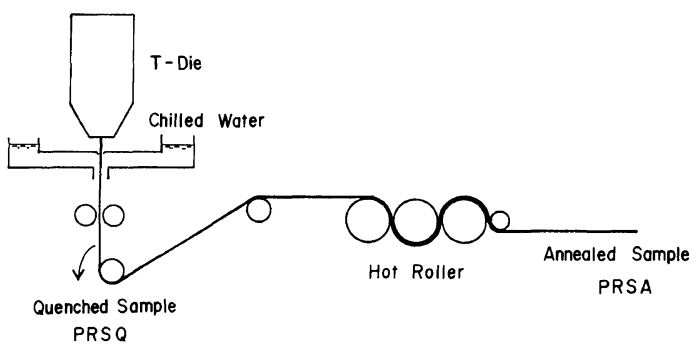

Figure 1. Schematic representation of PURELAY preparation.

called PURELAY were supplied by Idemitsu Petrochemical Co., Ltd. ${ }^{11}$ These films were prepared by extruding molten PP through a T-die, quenching them with flowing water, followed by roll annealing as shown in Figure 1. Two kinds of samples, PRSQ and PRSA, were taken in the manufacturing process. PRSQ was taken after water quenching, and PRSA (a commercial product) was obtained after the roll annealing process. The original PP pellets have the number and weight average molecular weights of 5800 and $407000 \mathrm{~g} \mathrm{~mol}^{-1}$, respectively, and melt index of 2 to $3 \mathrm{~g} / 10 \mathrm{~min}$. The details of the film preparation are described elsewhere. ${ }^{11}$ The reference PP films (PPR), which were also supplied by the company, were prepared from the same material but cooled with a chill-roll kept at $60-80^{\circ} \mathrm{C}$. In each sample, the film thickness was close to $300 \mu \mathrm{m}$.

\section{Turbidity Measurement}

Turbidity of PP films were measured with a ultraviolet and visible light spectrometer, Shimadzu UV-160. In order to reduce the contribution of the surface reflection and surface roughness of the sample, the sample films were immersed in $p$-xylene which has a roughly same refractive index, $n=1.4981$, to the sample.

\section{Light Scattering}

Light scattering patterns were measured photographically and photometrically. An LPC system, i.e., a light scattering measuring 
system having a linear image sensor developed in this laboratory, was used for the photometrical measurement. It has a photo-diode array of 512 pixels (MN512K, Matsushita Electronic Co., Ltd.) having a spatial resolution of $28 \mu \mathrm{m}$. The details of the system was reported elsewhere. $^{14}$

\section{Polarized Optical Microscopy}

Polarized optical microscopy was conducted with a Nikon polarized microscope. The spherulite size and number distributions were also measured with the microscope by changing the focal spot discretely from the top surface to bottom of the sample film. The depth resolution, i.e., the focal depth, was $1.2 \mu \mathrm{m}$.

\section{Densitometry}

Mass density variation was measured for sliced samples of $c a .30 \mu \mathrm{m}$ thick with a density gradient tube. The sliced films were prepared by cutting PP films glued on a thick PP plate with a microtome.

\section{RESULTS AND DISCUSSION}

\section{Turbidity}

Figure 2 shows the transmittance spectra of PRSQ, PRSA, and PPR. As shown in the figure, there is no characteristic absorption band in the range of wavelength from 300 to $700 \mu \mathrm{m}$. PRSQ and PRSA have significantly higher transmittance than PPR. It is interesting to note that the transmittance of PRSA (the annealed film) is higher than that of PRSQ (the quenched film) because generally most of semicrystalline polymers decrease their optical transmittance by annealing. This indicates that the transmittance is improved by annealing. The superiority in transmittance of PRSA is well characterized in terms of turbidity. Since no characteristic absorption band was observed in this wavelength range and the surface reflection was minimized by a refractive-index matching with $p$-xylene, most of the attenuation of the intensity of the incident light may result from light scattering. Therefore we define the turbidity, $\tau$, by the following equation,

$$
\tau=\log \left(I_{0} / I\right) / t
$$

where $I_{0}, I$, and $t$ are the incident and transmitted intensities and the sample thickness, respectively. Equation (1) is the same to the definition of absorbance. In this case, however, it is a measure of turbidity or haze because no characteristic absorption bands is present in this wavelength region and a comparison is done among the films composed of the same materials. Table I shows the char-

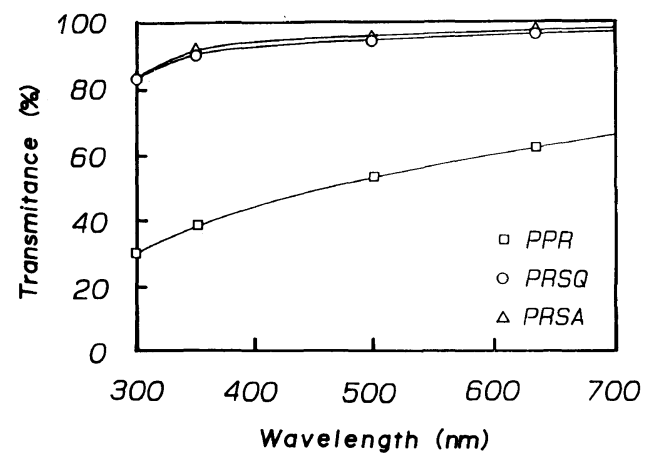

Figure 2. Variation of PPR, PRSQ, and PRSA transparency with wavelength.

Table I. Characterization of polypropylene films

\begin{tabular}{|c|c|c|c|c|c|}
\hline \multirow{2}{*}{$\begin{array}{c}\text { Sample } \\
\text { code }\end{array}$} & Density & \multirow{2}{*}{$\begin{array}{l}\text { Refractive } \\
\text { index }\end{array}$} & \multirow{2}{*}{$\begin{array}{c}\text { Birefringence } \\
\times 10^{3}\end{array}$} & \multirow{2}{*}{$\frac{\text { Melting point }}{{ }^{\circ} \mathrm{C}}$} & \multirow{2}{*}{$\frac{\text { Turbidity }}{\mathrm{cm}^{-1}}$} \\
\hline & $\mathrm{g} / \mathrm{cm}^{-3}$ & & & & \\
\hline PRSQ & 0.885 & 1.4914 & 1.75 & 161.5 & 0.35 \\
\hline PRSA & 0.898 & 1.4985 & 3.29 & 161.5 & 0.14 \\
\hline PPR & 0.898 & 1.4956 & 1.09 & 163.0 & 5.00 \\
\hline
\end{tabular}


acteristics of the three samples. PPR and PRSA have the same density, whereas PRSQ has a lower density and a lower refractive index than PRSA. This indicates that PRSQ has a lower degree of crystallinity than PPR and PRSA. The highest value in birefringence for PRSA is due to the roll annealing process. The highest melting temperature of PPR means that PP crystals are much developed in PPR than PRSQ and PRSA. The turbidity difference is significant among the three samples. The turbidity is dependent on the wavelength. Therefore we fixed the wavelength to be $632.8 \mathrm{~nm}$, which is the wavelength of a $\mathrm{He}-\mathrm{Ne}$ laser, so as to be consistent with the discussion based on the result of the light scattering experiment. The reasons of the lower turbidity of PRSA compared with PRSQ will be discussed in the following section.

\section{Light Scattering}

Figure 3 shows the $H_{\mathrm{v}}, V_{\mathrm{v}}$, and $H_{\mathrm{h}}$ scattering intensity patterns for as prepared PPR, PRSQ, and PRSA. MD in the figure indicates the machine direction. Note the scales are different between PPR and others. At a first glance, all of the three samples seem to have a spherulitic texture since they show a typical four leaf patterns in the $H_{\mathrm{v}}$ scattering patterns. However the maximum azimuthal angle where the scattering intensity has an optimum, $\mu_{\max }$, is away from $45^{\circ}$ for PRSQ and PRSA, where the azimuthal angle is taken with respect to MD as shown in Figure 4. The value of $\mu_{\max }$ was carefully determined photometrically with the LPC system when the sample was rotated in the plane perpendicular to the incident light. This trend is more clearly seen in Figure 4 which compares the $H_{\mathrm{v}}$ scattering patterns for the as-prepared (upper) and annealed $\left(160^{\circ} \mathrm{C}\right.$ for one hour) films (lower). P. and A. in the schematically drawn figure at the upper-left corner indicate the directions of the polarizer and analyzer, respectively. In the case of PPR,

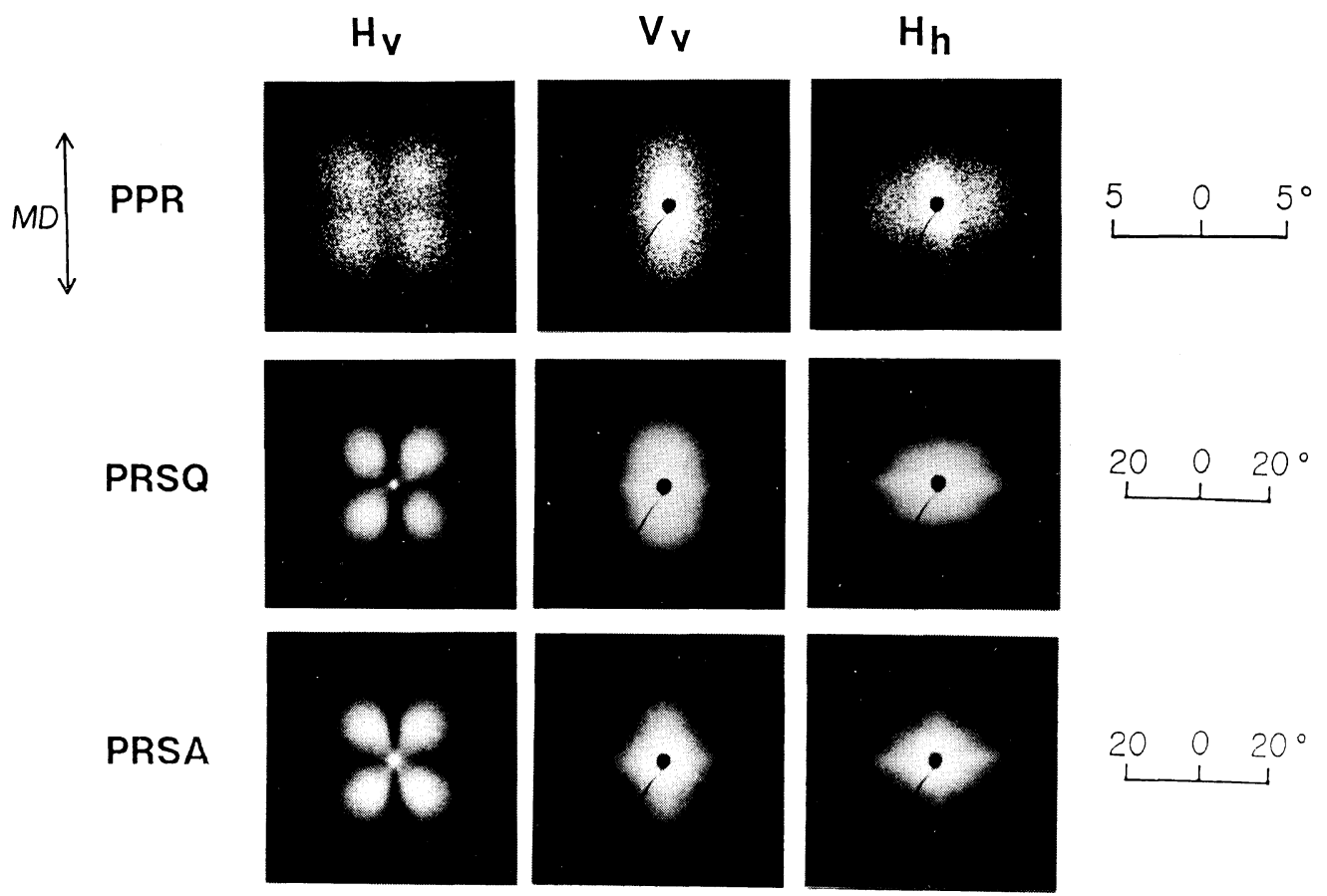

Figure 3. $H_{\mathrm{v}}, V_{\mathrm{v}}$, and $H_{\mathrm{h}}$ scattering patterns for as prepared PPR, PRSQ, and PRSA films. MD indicates the machine direction. 


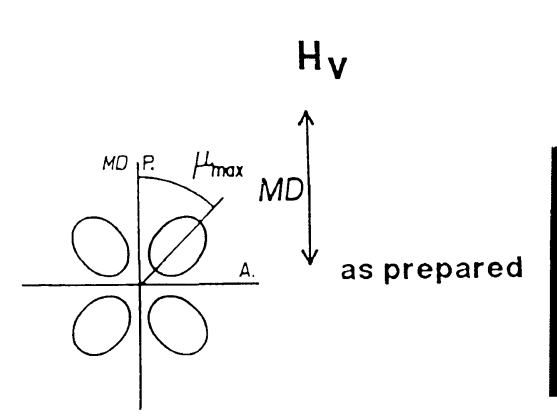

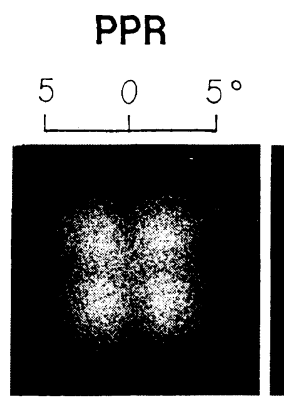

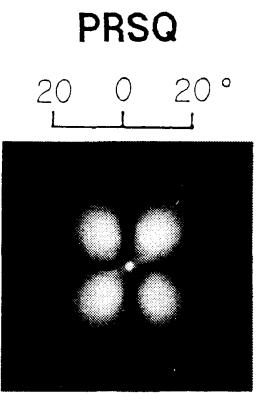

40

2.43

11.0

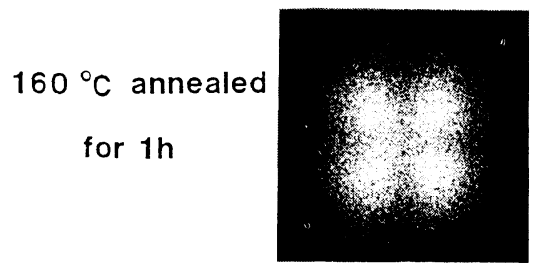

$\mu_{\max }($ deg. $) \quad 45$

$\mathrm{R}(\mu \mathrm{m})$

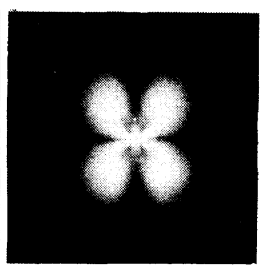

32
PRSA

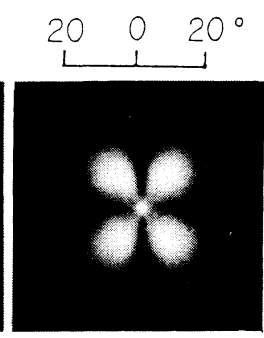

41

2.75

Figure 4. $H_{\mathrm{v}}$ light scattering patterns for PPR, PRSQ, and PRSA. P. A. and MD denote the directions of the polarizer, analyzer, and the machine direction, respectively. $\mu_{\max }$ and $R$ denote the azimuthal angle at peak maximum and the average radius of the spherulites or sheaves.

the shape of the scattering patterns does not change by annealing, and $\mu_{\max }$ remains to be $45^{\circ}$. PRSQ and PRSA change, however, their patterns by annealing and $\mu_{\max }$ moves from $40^{\circ}$ to $32^{\circ}$ and $41^{\circ}$ to $37^{\circ}$, respectively for PRSQ and PRSA. The average radius of the spherulite, $R$, was estimated with the Stein equation $^{15}$;

$$
4 \pi\left(R / \lambda^{\prime}\right) \sin \left(\theta_{\max } / 2\right)=4.09
$$

where $\lambda^{\prime}$ and $\theta_{\max }$ are the wavelength of the light in the medium and the scattering angle at peak maximum, respectively. The values of $R$ for PPR is about 5 times larger than those for PRSQ and PRSA. By annealing, $R$ increases for PRSQ and PRSA whereas that for PPR remains constant. Another interesting feature seen in the figure is the appearance of the superstructure by annealing. An additional scattering pattern of an "asterisk-shape" appeared around the origin of the scattering patterns for the annealed films. The appearance of $\mu_{\max }$ away from $45^{\circ}$ in PRSQ and PRSA may indicate that PRSQ and PRSA have deformed spherulites or sheaf-like structures. ${ }^{16,17}$ If one assumes that the films consist of spherulites, the light scattering patterns indicate that the spherulites are deformed along the direction perpendicular to the machine direction (MD), e.g., by 1.2 times in the case of $\mu_{\max }=40^{\circ}$, according to the spherulite deformation model proposed by Nomura $e t$ $a l .^{18}$ If this is the case, these deformed spherulites were formed by crystallization in a flow field during the production process. In this case, however, the asterisk patterns appeared in the annealed PRSQ and PRSA can not be explained. The sheaf-like structure, which is 

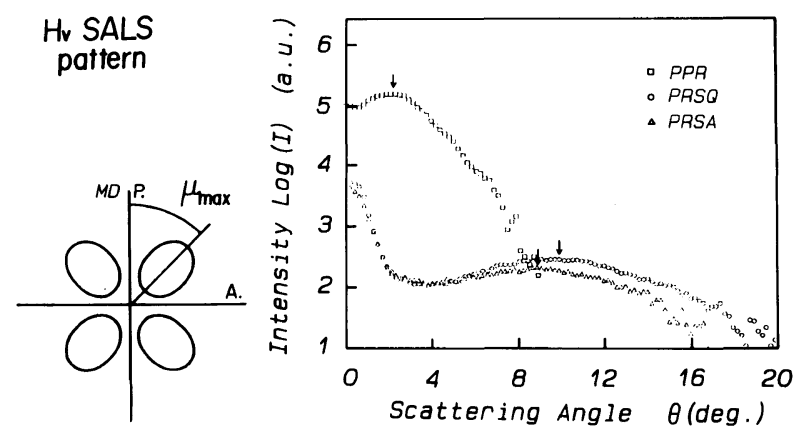

Figure 5. Variation of the $H_{\mathrm{v}}$-light scattering intensity distribution curves for PPR, PRSQ, and PRSA.

another candidate to explain Figures 3 and 4, is often found in row nucleated crystals, e.g., tubular extruded polybutene-1. ${ }^{16,17}$ Hashimoto et al. ${ }^{17}$ observed a similar asterisk pattern in the polybutene- 1 film at the low angle region and successfully explained this pattern via a row-nucleated sheaf model. Takahashi employed the model to simulate the scattering patterns of PRSQ and PRSA. ${ }^{19}$ In the case of high transparent PP films, such row nucleated crystals may be formed by temperature gradient and/or stretching during the film preparation. In both cases, the crystallization took place selectively in the transverse direction. i.e., the direction perpendicular to MD rather than along MD due to the flow field and/or temperature gradient along MD. If we use the Stein's equation ${ }^{17}$ for the relation between spherulite radius and the scattering angle at peak maximum, the average radii are calculated to be $11,2.43$, and $2.75 \mu \mathrm{m}$ respectively for as-prepared PPR, PRSQ, and PRSA and 11.0, 2.61, 2.85 $\mu \mathrm{m}$ for the annealed PPR, PRSQ, and PRSA, respectively.

Figure 5 shows $H_{\mathrm{v}}$ scattering intensity distributions for PPR, PRSQ, and PRSA. As shown in the figure, the magnitudes of the scattering intensities at maximum, $I_{\mathrm{m}}$, indicated with arrows, are remarkably different between PPR and PRS (PRSQ and PRSA). $I_{\mathrm{m}}$ for PPR is $\mathrm{ca} .10^{3}$ times stronger than those for PRSQ and PRSA. The difference in the scattering intensity between PRSQ and PRSA is also seen

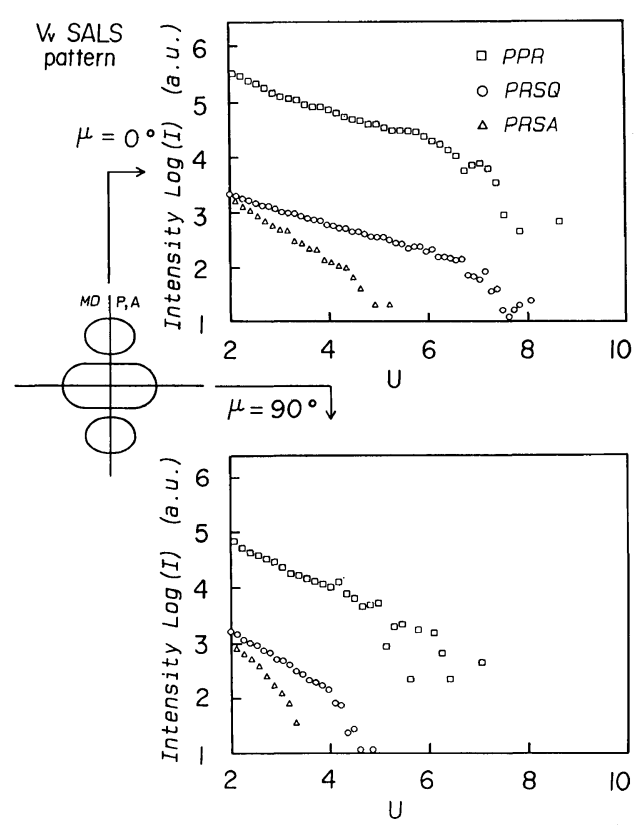

Figure 6. Variation of the $V_{\mathrm{v}}$-light scattering intensity distribution curves for PPR, PRSQ, and PRSA. $U$ is the reduced scattering vector defined by $U=4 \pi\left(R / \lambda^{\prime}\right)$ $\sin (\theta / 2)$.

in the figure.

Figure 6 shows the $V_{\mathrm{v}}$ scattering intensity variations for PPR, PRSQ, and PRSA in the directions along and perpendicular to MD. The abscissa is normalized to $U$ with respect to the average spherulite radius $R$, i.e., $U=$ $4 \pi\left(R / \lambda^{\prime}\right) \sin (\theta / 2)$, where $\theta$ is the scattering angle. The lower $U$ region than $U=2$ is eliminated here since an effect of stray scat- 
Table II. Quantitative comparison of the scattering intensity

\begin{tabular}{|c|c|c|c|c|}
\hline $\begin{array}{l}\text { Polarization } \\
\text { condition }\end{array}$ & $\mu$ (deg.) & $\begin{array}{l}\text { Sample } \\
\text { code }\end{array}$ & $\begin{array}{l}\text { Intensity } \\
\text { (a. u.) }\end{array}$ & $\begin{array}{l}\text { Intensity } \\
\text { ratio }\end{array}$ \\
\hline \multirow{3}{*}{$H_{\mathrm{v}}$} & \multirow{3}{*}{$\mu_{\max }$} & PPR & 15622 & $1 \quad(1)$ \\
\hline & & PRSQ & 29 & $1 / 539(1 / 93)$ \\
\hline & & PRSA & 22 & $1 / 710(1 / 64)$ \\
\hline \multirow{7}{*}{$V_{\mathrm{v}}$} & \multirow{3}{*}{$\mu=0^{\circ}$} & PPR & 353240 & 1 \\
\hline & & PRSQ & 2160 & $1 / 164$ \\
\hline & & PRSA & 1803 & $1 / 196$ \\
\hline & & & & \\
\hline & \multirow{3}{*}{$\mu=90^{\circ}$} & PPR & 78000 & 1 \\
\hline & & PRSQ & 1716 & $1 / 45$ \\
\hline & & PRSA & 1054 & $1 / 74$ \\
\hline \multirow{7}{*}{$H_{\mathrm{h}}$} & \multirow{3}{*}{$\mu=0^{\circ}$} & PPR & 249689 & 1 \\
\hline & & PRSQ & 1098 & $1 / 227$ \\
\hline & & PRSA & 1402 & $1 / 178$ \\
\hline & \multirow{4}{*}{$\mu=90^{\circ}$} & & & \\
\hline & & PPR & 119465 & 1 \\
\hline & & PRSQ & 2897 & $1 / 41$ \\
\hline & & PRSA & 1910 & $1 / 63$ \\
\hline
\end{tabular}

tering was significantly high. Figure 6 also shows the scattering intensity from PRSA is the lowest.

A quantitative comparison of the scattering intensities between the three samples is shown in Table II. For the $H_{\mathrm{v}}$ patterns, the scattering intensities at peak maximum, $\mu_{\max }$, were compared to each other. The estimated intensity ratios (without parenthesis) are compared with the calculatrd values (with parenthesis) based on the relation;

$$
\begin{gathered}
I \sim R^{3} \text { (for a volume-filled-spherulite } \\
\text { system) }
\end{gathered}
$$

The observed intensity ratios are much smaller than the calculated ones based on an assumption of a volume-filled spherulite system without change in the principal polarizabilities of the spherulite. This indicates that the intensity difference in PPR, PRSQ, and PRSA cannot be accounted for only by the change in the spherulite radius. It is interesting to note that the maximum scattering intensity, $I_{\mathrm{m}}$, is larger for PRSA than that for PRSQ, although

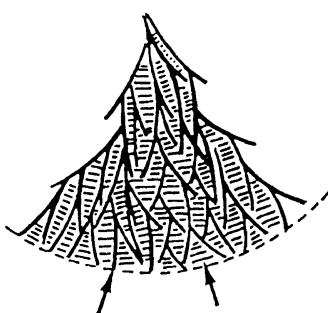

\section{Radial Cross-hatched lamellae lamellae}

Figure 7. Schematic representation of crosshatched lamellae.

the average radius of spherulites of PRSA is larger than that of PRSQ. This tendency can also be seen in the results for the $V_{\mathrm{v}}$ and $H_{\mathrm{h}}$ scattering where the intensity was compared at $U=2$ and at either $\mu=0^{\circ}$ or $90^{\circ}$. The comparison at lower $U$ could not be done because the presence of some stray scattering in the $V_{\mathrm{v}}$ and $H_{\mathrm{v}}$ scattering patterns at low angles. These results also show that PRSA has the lowest scattering intensity among the three samples, indicating that PRSA is clearer than PRSQ. Therefore PRSA may have a lower difference in polarizabilities between the spherulites and the surrounding matrix as well as a lower anisotropy in polarizability in a spherulite.

Norton et al. ${ }^{10}$ reported that crosshatched lamellae were grown epitaxially on the radially formed crystal lamellae as the secondary crystallization, as shown in Figure 7, when PP was annealed. The same phenomenon may happen when PRSQ is annealed, resulting in lowering of optical anisotropy in polarizability.

\section{Optical Microscopy by Stage Rotation}

In general, a spherulite does not change its view under a polarizing microscope (POM) by rotating the sample or the polarization direction. Keith classified the spherulite of isotactic polypropylene into five categories. ${ }^{8}$ According to the classification, PPR seems to belong to Type III. In the case of PRSQ or 

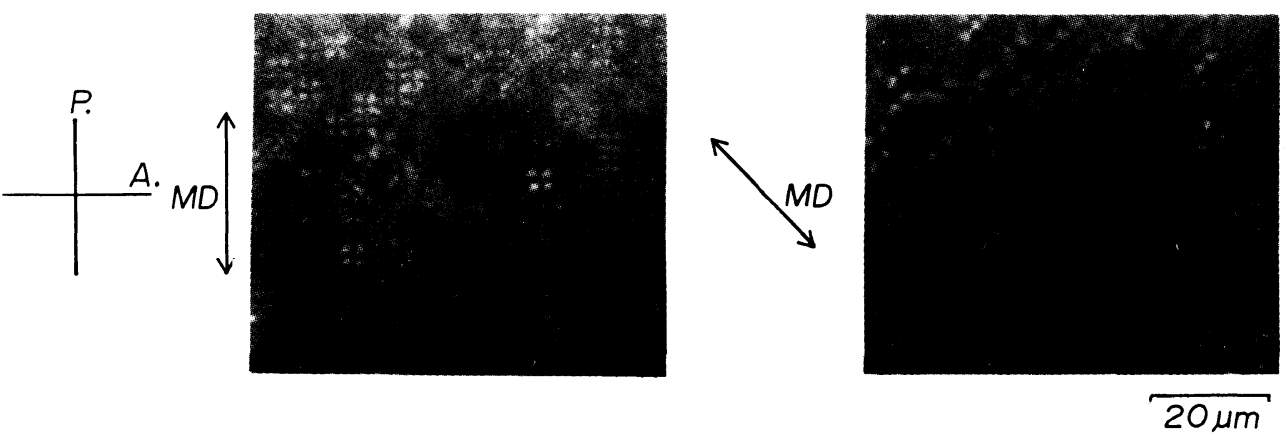

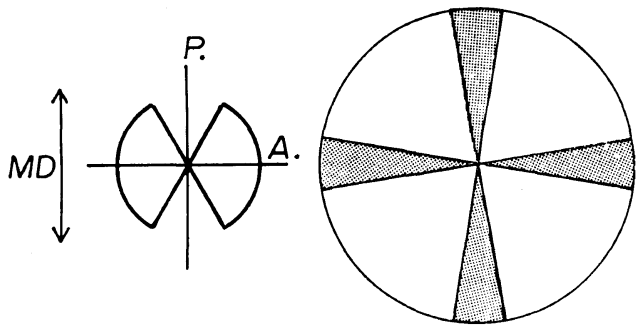

(A)
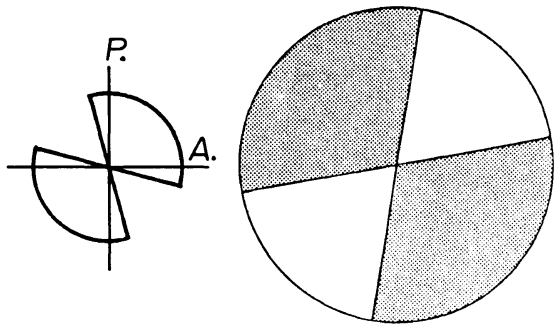

(B)

Figure 8. Polarized micrographs for PRSQ films. MD is along (A) and tilted by $45^{\circ}$ (B) with respect to the polarization direction $\mathrm{P}$. A. indicates the direction of the analyzer. By rotating the sample stage, the four leaf patterns change to a two-leaf pattern, indicating the presence of the sheaf-like structure as schematically shown in the lower portion of the figure.

PRSA, however, the POM pattern depends on $\mathrm{MD}$ and the polarization direction. Figure 8 shows the POM patterns of PRSQ under the extinction condition (MD is parallel to the direction of polarizer, P.) (A) and under the diagonal condition (MD is diagonal with respect to $\mathrm{P}$. and $\mathrm{A}$.). In the case of (A), a maltese cross pattern can be seen whereas the case (B) shows two-leaf patterns. This phenomenon can be explained with a sheaf-like model as follows: Since a sheaf has a structure of a two-leaf (two sectors connected diagonally at their origins), any crystalline structure is absent in the direction perpendicular to the sectors. If a sheaf is placed like (A), it shows a four leaf pattern since the extinction directions are vertical and horizontal. However, if it is placed like (B), it shows a two-leaf pattern since the absence of the crystalline structure in MD as shown in (B). A similar phenomenon was observed in PRSA.
The change in the view by stage rotation, however, can be also explained by assuming that the object is present in an optically anisotropic media. ${ }^{20}$ Although a direct observation in terms of a transmission electron microscope was tried by Iwamoto, any clear evidence of the sheaf-like structure was not obtained. ${ }^{21}$ Therefore we cannot conclude the presence of the sheaf-like structure in PRSQ and PRSA at this stage. The sheaf-like structure, however, seems to be a reasonable model to account for the observed results by light scattering and by optical microscopy.

\section{Observation of Cross Sections with an Optical Microscope}

Figure 9 shows the polarized optical micrographs of the cross sections of PPR, PRSQ, and PRSA. In the case of PPR, spherulites are filled in the entire range of the cross section. Contrary to this, the spherulite 
PPR

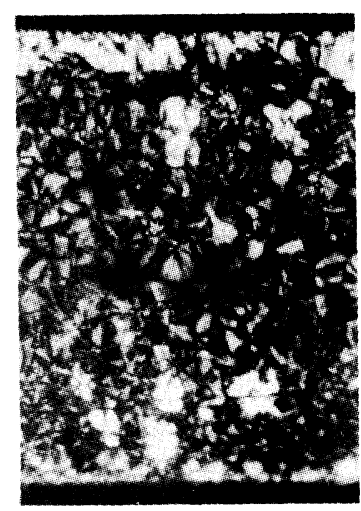

PRSQ

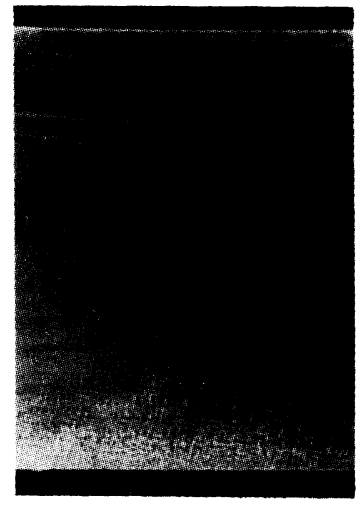

PRSA

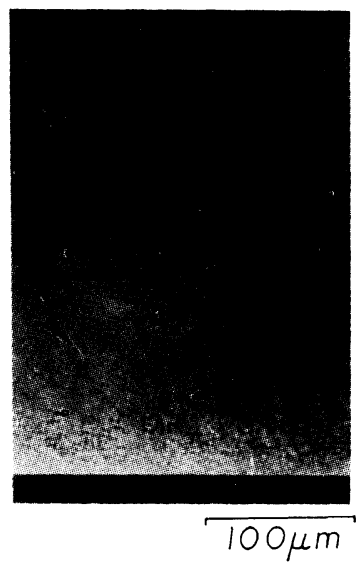

Figure 9. Cross polarized photographs of cross sections of PPR, PRSQ, and PRSA films.

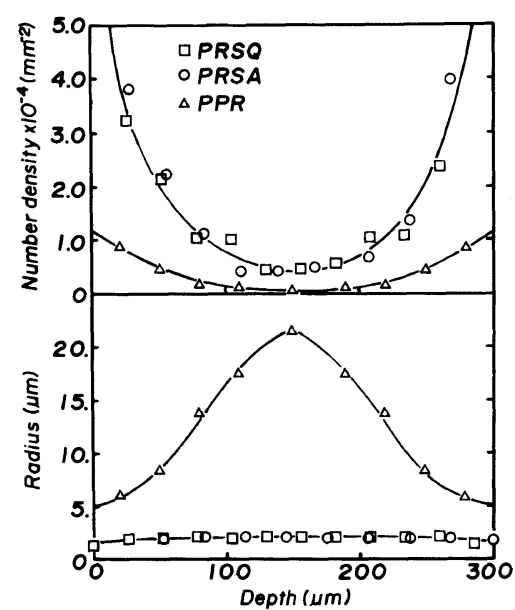

Figure 10. Depth profiles of the number density and radius of spherulites for PPR, PRSQ, and PRSA.

(or sheaf-like structure) sizes are remarkably small for PRSQ and PRSA and those populations are localized at the film surface and almost no distinct structure is seen in the middle part of the cross section.

Figure 10 shows the depth profilings of the number density and radius of the spherulites (or sheaf-like structure) for PPR, PRSQ, and PRSA. In the case of PPR, the radius increases with the depth according to lowering of the number, keeping the volume filling in the system. Contrary to this, a characteristic feature is seen in the depth profiles in PRSQ and PRSA. Although the number density variation with depth for PRSQ and PRSA is much steeper than that for PPR, the radius is invariant with the film depth. The volume fraction of spherulites of PRSQ and PRSA estimated from Figure 10 was $\mathrm{ca}$. 20\%. Since the degree of crystallinity is much higher than $20 \%$, a large number of invisible crystals, e.g., micro-crystallites are present in addition to the spherulites which were observed with an optical microscope.

\section{Mass Density Variation with Depth}

Figure 11 shows the density variation with film depth. It is interesting to note that only PRSQ has depth dependence of density. PPR seems to have well developed crystals along its film thickness direction, which is consistent with the result of Figure 10, i.e., the presence of volume filled spherulites. In the case of PRSA, the crystallinity may be improved by the roll annealing process particularly in the middle part of the film, without growing in radius of the spherulites. Homogenization of densities along the film thickness direction may be one of the major reasons why PRSA has a 


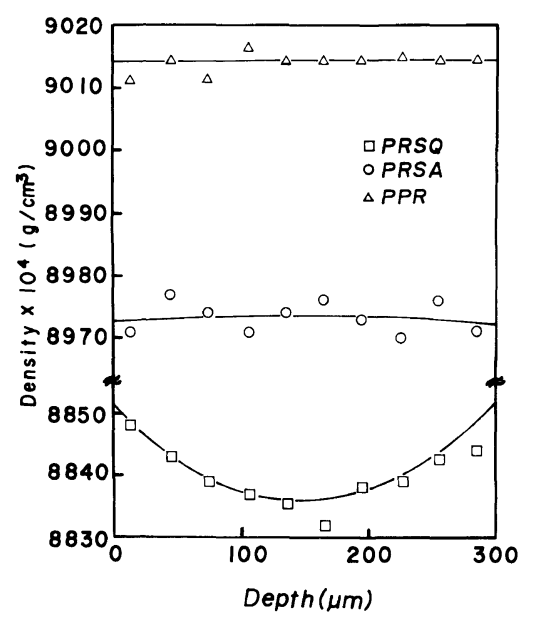

Figure 11. Depth profils of mass density for PPR, PRSQ, and PRSA.

lower turbidity than PRSQ.

\section{CONCLUSION}

Superstructures of high transparent polypropylene films PRSQ and PRSA, were investigated by means of light scattering, optical microscopy, and densitometry. The high transparency results mainly from the smaller size of spherulites of PRSQ and PRSA than the reference PP films. The transparency was improved by a roll annealing process done on PRSQ, which was explained by developing of crosshatch lamellae which played an important role in reducing the anisotropy of polarizability of spherulites. The sheaf-like structure seems to be a reasonable model to account for the observed results by light scattering and by optical microscopy.

The number density and size of spherulites (or sheaf like structures) and mass density were measured along their thickness direction. The density homogenization was attained by annealing whereas the number density and size of the spherulites remained unchanged. This may be the another reason for the improve- ment in transparency by annealing, which is characteristic to polypropylene.

Acknowledgment. We are grateful to Packaging Products R \& D Center, Plastics Products Department, Idemitsu Petrochemical Co., Ltd., Himeji 672, for supplying polypropylene films studied in this work.

\section{REFERENCES}

1. H. Awaya, Kobunshi, 14, 379 (1965).

2. A. T. Jones, J. M. Aizlowod, and D. R. Bechett, Makromol. Chem., 75, 133 (1964).

3. E. J. Addink and J. Beitema, Polymer, 2, 185 (1961).

4. G. Natta and P. Corradini, Nuovo Cimento (Suppl.), 15, 40 (1960).

5. H. D. Keith and F. J. Paden, J. Appl. Phys., 30, 1485 (1960).

6. G. Shi, B. Huang, and J. Zhang, Makromol. Chem. Rapid Commun., 5, 573 (1984).

7. G. Zhou, Z. He, J. Yu, Z. Han, and G. Shi, Makromol. Chem., 187, 633 (1986).

8. F. J. Padden, Jr. and H. D. Keith, J. Appl. Phys., 30, 1479 (1959).

9. D. C. Bassette and R. H. Olley, Polymer, 25, 935 (1984).

10. D. R. Norton and A. Keller, Polymer, 26, 704 (1985).

11. Jpn. Patent, S58-203018 (1983).

12. M. Shibayama, K. Imamura, K. Katoh, and S. Nomura, J. Appl. Polym. Sci., 42, xxxx (1991).

13. M. Shibayama, K. Katoh, and S. Nomura, in preparation.

14. H. Katoh, Master dissertation submitted to Kyoto Institute of Technology (1987).

15. R. S. Stein and M. B. Rhode, J. Appl. Phys., 31, 1873 (1960).

16. T. Hashimoto, A. Todo, Y. Murakami, and $\mathrm{H}$. Kawai, J. Polym. Sci., Polym. Phys. Ed., 15, 701 (1977).

17. T. Hashimoto, A. Todo, and H. Kawai, Polym. J., 10, 521 (1978).

18. S. Nomura, M. Matsuo, and H. Kawai, J. Polym. Sci., Polym. Phys. Ed., 12, 1371 (1974).

19. D. Takahashi, Master dissertation submitted to Kyoto Institute of Technology (1988); D. Takahashi, K. Katoh, M. Shibayama, and S. Nomura, Pep. Prog. Polym. Sci. Jpn., 31, 179 (1988).

20. F. Khoury, private communication.

21. T. Iwamoto, Master dissertation submitted to Kyoto Institute of Technology (1989). 